\title{
Design and Implementation for Sports Physical Fitness Analysis and Evaluation of Decision System
}

\author{
J.Z. HAN \\ Institute of Physical Education, University of Jinan, physical education, Shandong, China
}

M. X. LI

School of Nursing, Shandong University of Traditional Chinese Medicine, Nursing, Shandong, China

L. YANG

Institute of Physical Education, University of Jinan, physical education, Shandong, China

\begin{abstract}
The paper designs and realizes an analysis and evaluation of decision system about the effect of sports on physical fitness. It puts forward a decision mechanism based on the agent system which connects Internet technology and information technology by analyzing deficiencies such as disparity of traditional decision mechanism. It also designs the system architecture of whole system on the basis of analyzing basic system thoughts and structures. Thereinto, it does a detailed design to the tool structure of physical fitness analysis, the processing method of index data, the cooperative control mechanism and reasoning mechanism. The tool structure of physical fitness analysis is divided into: the perception and communication module, the problem analysis and solving module, the knowledge system module and the knowledge base's management module. Finally, through a practical test, it analyzes the relationship between data volume and decision time and precision rate in detail. It is found through a lot of tests that: an increase of decision precision rate by raising data volume can largely provide system's work efficiency indeed, effectively shorten the decision time and greatly raise the decision precision rate.
\end{abstract}

KEYWORD: Sports; Decision System; Physical Fitness; System Structures; database

\section{INTRODUCTION}

With improvement of modern living conditions, people increasingly tend to do seldom sports. However, during a long-term sports process, the human body will change. Most sports can enhance human physical fitness and physical quality to a certain extent, yet excessive sports or unreasonable sports do harm to human bodies. However, the analysis and evaluation for physical fitness of person arteries and veins is a complicated system project, belonging to a non-structural strategic decision problem. Considering the traditional method which has a large coverage area of data, a long duration time and a large number of data volume and also involves many units and decision makers, because the decision process needs necessary information that has a quite large dispersed scale, so this kind of large-scale decision activity is inconvenient to be implemented in a centralized way, it should adopt a dispersed control accompanied by group decision makers to finish it. But the dispersed decision brings about many new issues such as insufficient data concentration and decreased decision precision rate. Therefore, by connecting modern information technology and computer technology, a scientific and reasonable analysis to human's physical fitness during a sports process can be done in order to make a corresponding decision to guide people to do reasonable sports that can effectively improve human's physical quality. To solve the above issues, there should have a revolutionary leap from technology. With rapid development of technologies such as dispersed artificial intelligence and Internet, an information system which provides support for group decision activities naturally appears. By constructing the analysis of students' physical fitness and the evaluation of group decision support system, the study is beneficial to promote the development of physical fitness research work and boost a new technological revolution of physical fitness research. In order to carry out the physical fitness research work in a deep-going way, the connection of modern information technology and computer technology should be done for the sake of do a research for relationship between human sports and physical fitness, and then technologies such as multi-agent, Internet and CS are applied to come up with a new physical fitness analysis and evaluation system. On the basis of doing large-scale data collection work, an online physical fitness analysis and evaluation of group decision support system is developed, offering a unified physical fitness analysis and evaluation software platform to people 
and meanwhile making physical fitness data resources shareable and increasing the decision mechanism's interactivity and reference data.

\section{SYSTEM DESIGN}

\subsection{Basic System Thoughts and Technical Analysis}

The application of technical means of modern computer science and network communication and the development of long-range multi-group decision support system facing the physical fitness analysis and evaluation of whole nation's students can fully utilize current various physical fitness analyses and evaluation materials, technology and expert knowledge, making more people available to share physical fitness data resources with the help of the system to reach scientificity and real-time performance of decision analysis. Hence, the system collects related technologies in different sites with management experts or software systems through Internet or local area networks of all departments to do online team work, thus spending minimum costs and expenses, using the latest materials and technologies in a fastest and most direct speed, and accurately making analyses and evaluations. The basic core technologies involved in the system development are: (1) The multi-agent technology. Agent, a basic term of dispersed artificial intelligence, refers to a kind of computational substance which can sense environment and do selfrule operation to realize a series of targets representing designers or users. It characterized with independence, communication, perception and dynamic capability. The multi-agent technology has become an important branch of dispersed intelligent control, applied to the solving function or complicated issues dispersed on geography, and made the solving of problem reliable and extensible in a high speed. The introduction of multi-agent to the system can realize functions such as coordination, surveillance and maintenance during a group decision process and provide intelligent decision support for the system. (2) The cooperative work technology supported by computer. It is used for the main support environment of online group decision, and belongs to a new cross field comprehensively made by many disciplines such as computer technology, communication engineering, system engineering, psychology and sociology. It studies on how to use relevant computer technologies to assist a group organization to accomplish common work.

\subsection{System Structures}

The whole system structure is constructed by three levels: the resource level, the resource management level and the human-computer interaction level, see figure 1. With respect to level structures: the resource level is mainly made up of database, knowledge base, rule base and model base; the resource management level consists of the management systems of four bases, and it is correlative among management systems in order to accurately, reliably and conveniently transfer resource; the human-computer interaction level actualizes a direct dialogue between the system and user. With respect to elements which construct the system, the system is made up of four subsystems, namely, database subsystem, knowledge base subsystem, rule base subsystem and module base

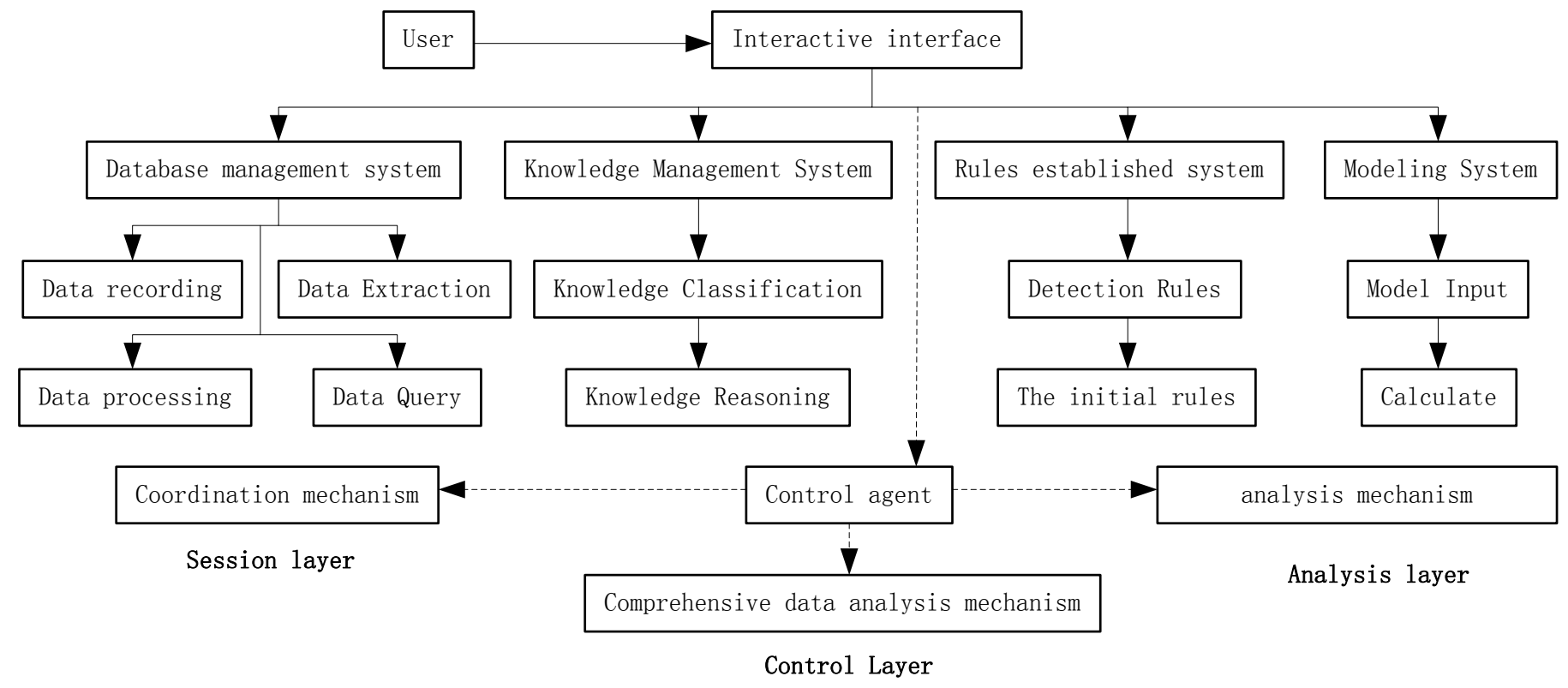

Figure 1 Structure design of physical fitness sports decision system 
subsystem. The system belongs to a group coordination decision support system facing dispersed computational environment under the network condition, its basic frame is divided into three levels. The dialogue level faces decision makers all over the country and consists of several interactive Agents. The interactive Agent, an intelligent interface to have a human-computer dialogue between user and computer, is responsible for receiving problem information that is essential to solve for users, and then transferring to the control Agent, and next inputting the final solving result to the corresponding user. The control level is made up of integration and control of two Agent and cooperative result database.

\section{REALIZATION OF DECISION SUPPORT SYSTEM}

\subsection{Structure Design of Physical Fitness Analysis Tool}

The problem solving is achieved by all physical fitness analysis Agent. Structures of all physical fitness analysis Agent are the same, including perception communication module, problem analysis and solving system and knowledge system, see figure 2. (1) The perception communication module. It, an interface part of physical fitness analysis Agent, presides over and controls Agent to communicate, obtains and comprehends status information related to problems under integrated environment, makes information changes with other physical fitness analysis Agent, and transfers analysis results, etc.. (2) The problem analysis solving module. It is a core of physical fitness analysis Agent, and can directly start up the knowledge base to do reasoning and indirectly drive database and model base to run. (3) The knowledge system module. The database is used for storing all data needed during a physical fitness analysis Agent process. The module base and module base management system. The module base is used for storing all modules of analysis decision, including modules of fuzzy mathematics class, statistics class, graphics class and decision. (4) The knowledge base management module. The knowledge base reserves expert knowledge of physical fitness analysis and evaluation and experience knowledge of selection analysis module. They can let the system automatically select a certain modules and method according to basic facts and data provided by decision makers, thus emerging concrete module cases accorded with requirements of decision makers and corresponding strategies in accordance with expert knowledge. The knowledge base management system is responsible for functions such as knowledge acquirement, searching and storage.

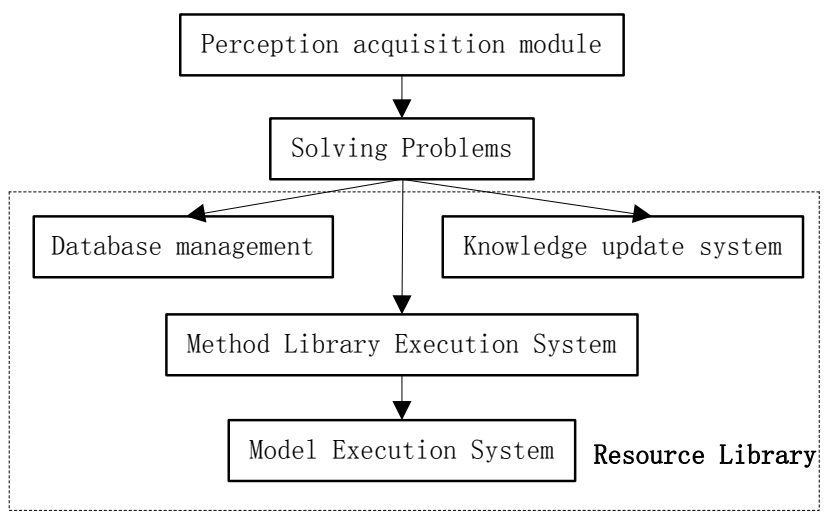

Figure 2 Physical fitness analysis function structure

\subsection{Design of Cooperative Control Mechanism}

It finishes the cooperative control task of system core mainly by control Agent. The control Agent consists of two parts--knowledge base and control. (1) The part of knowledge base: it stores certain knowledge needed in coordination and consists of strategic knowledge base, activity status base and resource base. There into, the strategic knowledge base describes the cooperative strategy to physical fitness analysis Agent (including methods and models); the activity status base records activity status of all physical fitness analysis Agent, including initialization, restart, hang-up, termination, activation, complement, running and rest time for running; the resource base records the consumption condition of resource by current activity. (2) The part of control: it is made up of cooperative reasoning machine, task execution controller and activity monitor. The coordination occurs when a single physical fitness analysis Agent of certain problem cannot be solved and then needs many of them to do cooperative solving. At this moment, the cooperative reasoning machine does reasoning to make sure which physical fitness analysis Agent can have group coordination at present according to targets of problem, functions of all physical fitness analysis Agent, running status and models and methods in the strategic knowledge base.

\section{REAL TEST OF DECISION SUPPORT SYSTEM}

The system is put into actual use after many tests and promotion. The result shows that system functions are complete and operation is stable, meeting needs of test crowd and realizing scientific guidance of health of person arteries and veins movement. Among them, the data input way means after the user's physical fitness test data is imported into the system by a card reader, the system automatically generates a physical fitness test record. And the most crucial point of test process is to verify the analysis capability of inputting data by 
system, then make a decision and finally check the decision accuracy. We conduct a large quantity of tests to data volume import and time and precision rate for a system to make a decision, and also study the relationship between the two. The data volume import cannot be computed by data size, it should consider to import the numbers of people because each person's physical fitness condition is different, so imported data indexes are also different. At the same time, the computation of decision precision rate is counted according to judge whether the diagnosed person physical fitness matches with the decision made by system. The test number of people is 200 in total. The figure 3 shows the relationship between imported data volume and decision time. And it reveals the relationship between imported data volume and decision precision rate. From figures, it can be found that, when the test number of people increases, the system decision time obviously shortens and the system decision precision rate continuously rose. Because of besides reference to materials of inside database, the system decisionmaking also refers imported data. Hence, with an increase of imported data, reference data also grows, and then the time of decision-making will shorten, so the precision rate will rise at any time.
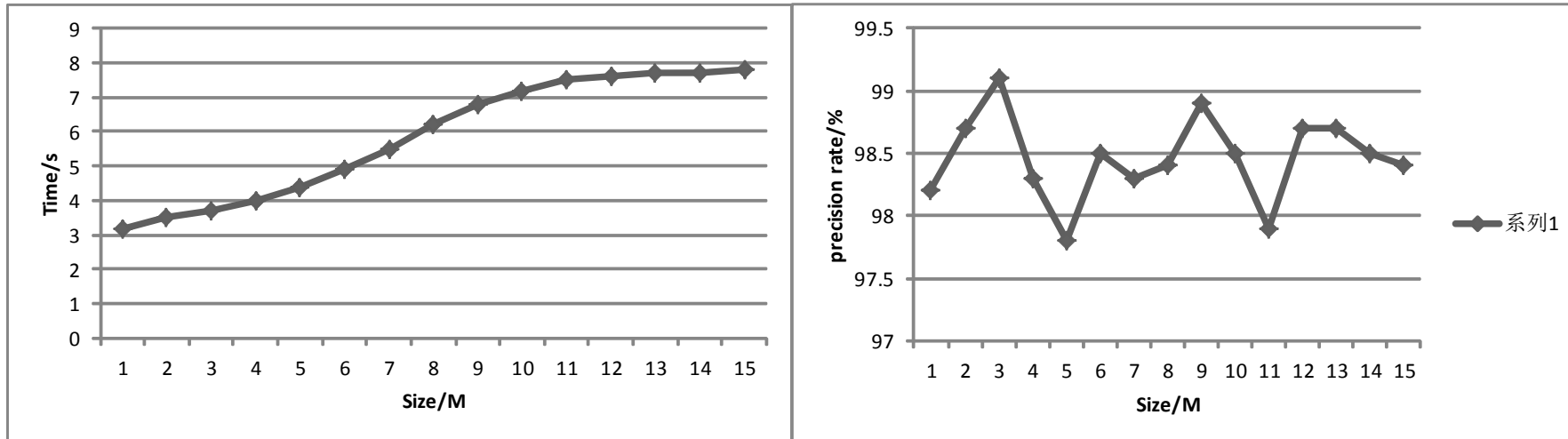

Figure 3 decision time and decision precision rate test result

\section{CONCLUSION}

The paper firstly analyzes certain previous achievements on research of sports to physical fitness, puts forward the thought to integrate data based on analyzing their deficiencies, introduces system structures and basic thoughts and then analyzes key technologies. Second, it designs the physical fitness analysis tool structure. The realization of system inside includes three parts, namely, treatment of index data, cooperative control mechanism design and decision recommendation mechanism design of data representation. It would be best to analyze relationship between imported data volume by system and decision time, and relationship between data volume and decision precision rate in detail through actual cases. Finally, the conclusion is made: the thought of increasing decision precision rate by adding imported volume of data is correct. The system can overcome deficiencies such as difficult data management and expansion caused by building a huge database in a centralized system, share data resources and exchange research achievements, reaching both decentralization and centralization. Thus it avoids repeated researches, improves work efficiency and has some practical values and reference values. The deficiencies of paper lie in that it has no single analysis to some special crowd. So the decision made by system cannot apply to all people. And the paper also has no detailed data collection mechanism to promote the data accuracy in itself. Therefore, the next work will focus on the data collection, design more reliable data collection nodes in order to increase the system decision accuracy.

\section{ACKNOWLEDGEMENT}

In this paper, the research was sponsored by Shandong Province Universities Science and Technology Fund (J13LK07)..

\section{REFERENCES}

[1] J.C.Yu, Z.X. Liu, J.Y.Tang, 2007. Transient electromagnetic detecting technique for water hazard to the roof of fully mechanized sub level caving face. Journal of China University of Mining \&Technology 36(4) 542 -546.

[2] Z.H. Jiang, J.H. Yue, S.C.Liu, 2007. Prediction technology of buried water- bearing structures in coal mines using transient electromagnetic method. Journal of China University of Mining and Technology 17 (2) 164 -167.

[3] Z.X. Liu, J.C.Yu, D. Guo, 2006. The application of the miningtransient electromagnetic method to the exploration of hydrological borehole. Geophysical and Geochemical Exploration 30(1) 59-61.

[4] C.Guo, B.Z. Liu, D.H. Bai, 2006. Prediction of water disasters ahead of tunneling in coal mine using continuous 
detection by TEMJ, Selismology and Geology 28 (3) 456462.

[5] Z.H.Jiang, J.H.Yue, S.C. Liu, 2007. Experiment of mine transient electromagnetic observation system of small multiturn coincident configuration. Journal of China Coal Society, 32(11) 1152-1 156.
[6] Z.X. Liu, S.C. Liu, Y.G.Liu, 2009. Research on transient electromagnetic field of mine water- bearing structure by physical model experiment. Chinese Journal of Rock Mechanics and Engineering, 28(2) 259 -266.

[7] B.Y.Jiang, 1992. Applid near zone magnetic source transient electromagnetic exploration. Geological Publishing House, Beijing. 